\title{
Ferromagnetic ordering in diluted magnetic semiconductors
}

\author{
A.H.Slobodskyy ${ }^{1,2,3}$, V.K.Dugaev ${ }^{1,4}$, M.Vieira ${ }^{4}$ \\ 1 Institute for Problems of Materials Science, \\ National Academy of Sciences of Ukraine, \\ 5 Vilde Str., 58001 Chernivtsi, Ukraine \\ 2 Institute of Physics, Polish Academy of Sciences, \\ al. Lotników 32/46, 02-668 Warszawa, Poland \\ 3 Physikalisches Institut, Universität Würzburg, Am Hubland, \\ 97074 Würzburg, Germany \\ 4 Department of Electronics and Communications, \\ Instituto Superior de Engenharia de Lisboa, \\ 1949-014 Lisbon, Portugal
}

Received November 22, 2001, in final form June 25, 2002

\begin{abstract}
We present a general approach to the problem of a ferromagnetic phase transition in diluted magnetic semiconductors. The Curie temperature of ferromagnetic transition is calculated in the mean field approximation. It is shown that the Curie temperature is determined by an integrated coupling between magnetic impurities.
\end{abstract}

Key words: magnetic semiconductors, magnetic impurities, ferromagnetism

PACS: $75.30 .-m, 75.50 . D d, 75.50 . P p$

\section{Introduction}

The study of the ferromagnetic ordering generated by carriers in doped semiconductors has been pioneered by experiments on PbMnSnTe compounds [1-3]. Recently this problem attracted much attention [4-10] in connection with possible applications of ferromagnetic semiconductors in spin electronics $[11,12]$. The most promising seems to be the GaMnAs semiconductor alloy with Curie temperature about $110 \mathrm{~K}$ [14]. This material has been proved to be an efficient injector of spinpolarized electrons with a long-time spin polarization.

The theory of ferromagnetism in diluted magnetic semiconductors has been presented by several groups. The main idea is that the ferromagnetic ordering is caused by the exchange interaction between magnetic impurities mediated by free electrons or holes $[4,15]$. This mechanism is commonly accepted now even though it does not 
explain the ferromagnetism at low density of carriers [10]. On the other hand, the theory of Dietl $[4,12]$ exploits a simplified model of the exchange interaction, the Zener model [16], which does not take into account the oscillating character of the RKKY interaction between magnetic impurities [17].

In a recent series of theoretical works, König et al. presented a mean field theory $[9,18]$, which starts from a system of free carriers interacting with magnetic impurities. Such an approach accounts for the effective exchange interaction at all distances, which is important for oscillating and/or long-range interactions. It also automatically incorporates three, four, etc. impurity couplings.

In the present work we develop a mean field theory, which is close to works $[9,18]$ but differs from it in several aspects. First, we treat the impurity magnetic moments classically, which is quite a good approximation for the impurities with a large moment. It permits to avoid the Holstein-Primakoff bosons, which make the problem nonlinear. The linearization within the description based on the HolsteinPrimakoff bosons was not justified in [9].

Here we develop a functional integral approach to the problem. Our approach can be a good starting point for a theory more sophisticated than a mean field approximation. It also permits to consider the disorder effects, which has been recently discussed in papers $[19,20]$.

\section{Model and general approach}

We start with the Hamiltonian of a free electron interacting with magnetic impurities

$$
H=-\frac{\hbar^{2} \nabla^{2}}{2 m^{*}}-J_{s d} \sum_{i} \boldsymbol{\sigma} \cdot \mathbf{M}_{i} \delta\left(\mathbf{r}-\mathbf{R}_{i}\right),
$$

where $J_{s d}$ is the $s$ - $d$ coupling constant, $\mathbf{M}_{i}$ is the magnetic moment of the $i$-th magnetic impurity, $\mathbf{R}_{i}$ is its position, $\boldsymbol{\sigma}=\left(\sigma_{x}, \sigma_{y}, \sigma_{z}\right)$, and $\sigma_{\alpha}$ are the Pauli spin matrices. Here, the first term is the Hamiltonian of free electrons in a semiconductor near the bottom of the conductivity band (and $m^{*}$ is the corresponding electron effective mass), and the second term describes the exchange interaction between an electron and the impurities. We assume that magnetic impurities are distributed randomly. The magnetic moments of the impurities $\mathbf{M}_{i}$ can be oriented arbitrarily, and our task is to find the thermodynamically favourable distribution of orientations of localized moments together with the magnetization of the electron gas. The Hamiltonian (1) acts on spinor wavefunctions of the electrons. The average concentration of magnetic impurities is denoted by $c$.

The Hamiltonian (1) can be rewritten in a second quantization form describing the electron gas in a degenerate semiconductor. We use the formalism for $T \neq 0$ :

$$
\begin{aligned}
H= & \int \mathrm{d}^{3} \mathbf{r} \psi^{\dagger}(\mathbf{r}, \tau)\left(-\frac{\hbar^{2} \nabla^{2}}{2 m^{*}}-\mu\right) \psi(\mathbf{r}, \tau) \\
& -J_{s d} \sum_{i} \int \mathrm{d}^{3} \mathbf{r} \psi^{\dagger}(\mathbf{r}, \tau) \boldsymbol{\sigma} \cdot \mathbf{M}_{i} \psi(\mathbf{r}, \tau) \delta\left(\mathbf{r}-\mathbf{R}_{i}\right),
\end{aligned}
$$


where $\psi(\mathbf{r}, \tau)$ is the spinor operator field in Heisenberg representation, $\tau$ is the imaginary (thermodynamic) time [21], and $\mu$ is the chemical potential. It permits to treat the problem with a many-particle Hamiltonian and to use such methods as Feynman diagrams or the functional integration techniques.

We consider the partition function of the system to be a functional integral [21] over Fermi fields $\psi(\mathbf{r}, \tau)$ and over all possible orientations of vector fields $\mathbf{M}_{i}$ (we assume the fixed absolute value $\left.\left|\mathbf{M}_{i}\right|=M\right)$

$$
Z=\int D \psi^{\dagger} D \psi \prod_{i} D \mathbf{M}_{i} \mathrm{e}^{-S}
$$

where $S$ is the action

$$
S=\int \mathrm{d} \tau \mathrm{d}^{3} \mathbf{r} \psi^{\dagger}(\mathbf{r}, \tau)\left(\frac{\partial}{\partial \tau}+H\right) \psi(\mathbf{r}, \tau)
$$

and the Hamiltonian $H$ is taken in the first quantization form of equation (1). It should be noted that the integration over Fermi fields implies that the $\psi, \psi^{\dagger}$ can be viewed as anticommuting (Grassmann) variables, for which the functional integration techniques has been developed [22].

\section{Free energy functional}

The direct averaging over the orientations of localized $\mathbf{M}_{i}$-fields, equation (3), makes the problem nonlinear in Fermi fields. To avoid this complication, we introduce some auxiliary fields. We can write the partition function using an additional integration over a vector field $\mathbf{m}(\mathbf{r})$

$$
Z=\int D \psi^{\dagger} D \psi \prod_{i} D \mathbf{M}_{i} D \mathbf{m} \delta\left[\mathbf{m}(\mathbf{r})-\sum_{i} \mathbf{M}_{i} \delta\left(\mathbf{r}-\mathbf{R}_{i}\right)\right] \mathrm{e}^{-S}
$$

with the following constraint equation

$$
\mathbf{m}(\mathbf{r})-\sum_{i} \mathbf{M}_{i} \delta\left(\mathbf{r}-\mathbf{R}_{i}\right)=0
$$

imposed by the $\delta$-function in equation (5).

The field $\mathbf{m}(\mathbf{r})$ has the meaning of the local magnetization field. In contrast to the $\mathbf{M}_{i}$-field, $\mathbf{m}(\mathbf{r})$ is defined at each point of space $\mathbf{r}$ and can fluctuate in both orientation and magnitude. The latter permits to avoid the hidden nonlinearity of $\mathbf{M}_{i}$-fields related to the constraint $\mathbf{M}_{i}^{2}=$ const.

As a next step, we use the Lagrange multiplier formalism to account for the constraint of equation (6). To this end, we integrate over an auxiliary vector field $\lambda(\mathbf{r})$

$$
\begin{aligned}
Z= & \int D \psi^{\dagger} D \psi \prod_{i} D \mathbf{M}_{i} D \mathbf{m} D \boldsymbol{\lambda} \exp \left[-S_{e}+\int \mathrm{d}^{3} \mathbf{r} \boldsymbol{\lambda}(\mathbf{r}) \cdot \mathbf{m}(\mathbf{r})\right. \\
& \left.-\sum_{i} \int \mathrm{d}^{3} \mathbf{r} \boldsymbol{\lambda}(\mathbf{r}) \cdot \mathbf{M}_{i} \delta\left(\mathbf{r}-\mathbf{R}_{i}\right)\right]
\end{aligned}
$$


where the electron part of the action is

$$
S_{e}=\int \mathrm{d} \tau \mathrm{d}^{3} \mathbf{r} \psi^{\dagger}(\mathbf{r}, \tau)\left(\frac{\partial}{\partial \tau}-\frac{\hbar^{2} \nabla^{2}}{2 m^{*}}-J_{s d} \mathbf{m}(\mathbf{r}) \cdot \boldsymbol{\sigma}\right) \psi(\mathbf{r}, \tau)
$$

As follows from equation (7), the field $\boldsymbol{\lambda}(\mathbf{r})$ can be associated with the local magnetic induction in the semiconductor.

The integration over fermion fields $\psi^{\dagger}$ and $\psi$ can be performed using the rules of Gaussian integration with the Grassmann numbers [22]

$$
\int D \psi^{\dagger} D \psi \mathrm{e}^{\psi \dagger A \psi}=\operatorname{det} A
$$

where $A$ is an arbitrary Hermitian matrix.

We can also average over the directions of the classical moments $\mathbf{M}_{i}$. Hereafter we obtain

$$
Z=\int D \mathbf{m} D \boldsymbol{\lambda} \mathrm{e}^{-\beta F}
$$

where

$$
-\beta F=\int \mathrm{d}^{3} \mathbf{r}\left[\operatorname{Tr} \sum_{n} \ln G^{-1}\left(-\mathrm{i} \varepsilon_{n} ; \mathbf{r}, \mathbf{r}\right)+\boldsymbol{\lambda}(\mathbf{r}) \cdot \mathbf{m}(\mathbf{r})+c \ln \left(\frac{\sinh [\lambda(\mathbf{r}) M]}{\lambda(\mathbf{r}) M}\right)\right],
$$

the electron Green function $G\left(-\mathrm{i} \varepsilon_{n} ; \mathbf{r}, \mathbf{r}^{\prime}\right)$ in the magnetization field $\mathbf{m}(\mathbf{r})$ obeys the following equation

$$
\left(-\mathrm{i} \varepsilon_{n}-\frac{\hbar^{2} \nabla^{2}}{2 m^{*}}-J_{s d} \mathbf{m}(\mathbf{r}) \cdot \boldsymbol{\sigma}\right) G\left(-\mathrm{i} \varepsilon_{n} ; \mathbf{r}, \mathbf{r}^{\prime}\right)=\delta\left(\mathbf{r}-\mathbf{r}^{\prime}\right)
$$

$c$ is the concentration of magnetic impurities, and $\beta=1 / T$ (here we measure $T$ in the energy units, $k_{\mathrm{B}}=1$ ). In equations (11) and (12) we used the Fourier transform from the thermodynamic time $\tau$ to the discrete Matsubara frequencies $\varepsilon_{n}=(2 n+1) \pi T$ for the Fermi fields [23].

We call $F$ the free energy functional in view of the analogy between the quantum field formulation of equations (10) and (11), and the conventional statistical theory.

\section{Saddle point equations}

The saddle point equations for the fields $\mathbf{m}(\mathbf{r})$ and $\boldsymbol{\lambda}(\mathbf{r})$, corresponding to an extremum of the free energy functional $F$ in equation (11), are found by calculating the variation of $F$ with respect to these fields

$$
\frac{\delta F}{\delta \mathbf{m}(\mathbf{r})}=0, \quad \frac{\delta F}{\delta \boldsymbol{\lambda}(\mathbf{r})}=0
$$

These equations acquire the following form

$$
-J_{s d} \operatorname{Tr} \sum_{n} \boldsymbol{\sigma} G\left(-\mathrm{i} \varepsilon_{n} ; \mathbf{r}, \mathbf{r}\right)+\boldsymbol{\lambda}(\mathbf{r})=0
$$




$$
m(\mathbf{r})+c M \operatorname{coth}[M \lambda(\mathbf{r})]-\frac{c}{\lambda(\mathbf{r})}=0 .
$$

Note that the latter equation relates absolute values of vector fields $\boldsymbol{\lambda}(\mathbf{r})$ and $\mathbf{m}(\mathbf{r})$ whereas their directions are the same.

In the limit of $\mathbf{m}(\mathbf{r}) \rightarrow 0$, we obtain from equations (14) and (15)

$$
\mathbf{m}(\mathbf{r})=-\frac{2 c M^{2} J_{s d}^{2}}{3} \sum_{n} \mathrm{~d}^{3} \mathbf{r}^{\prime} G_{0}\left(-\mathrm{i} \varepsilon_{n} ; \mathbf{r}, \mathbf{r}^{\prime}\right) \mathbf{m}\left(\mathbf{r}^{\prime}\right) G_{0}\left(-\mathrm{i} \varepsilon_{n} ; \mathbf{r}^{\prime}, \mathbf{r}\right)
$$

and

$$
\lambda(\mathbf{r})=-\frac{3 m(\mathbf{r})}{c M^{2}},
$$

where $G_{0}\left(-\varepsilon_{n} ; \mathbf{r}, \mathbf{r}^{\prime}\right)$ is the electron Green function for zero magnetization field in the Matsubara techniques.

Equation (16) can be solved by Fourier transformation over the coordinates, and we obtain

$$
\mathbf{m}(\mathbf{q})=-\frac{c M^{2} J_{s d}^{2}}{3 T} \Pi_{0}(\mathbf{q}) \mathbf{m}(\mathbf{q})
$$

where

$$
\Pi_{0}(\mathbf{q})=2 T \sum_{n} \int \frac{\mathrm{d}^{3} \mathbf{k}}{(2 \pi)^{3}} G_{0}\left(-\mathrm{i} \varepsilon_{n}, \mathbf{k}+\frac{\mathbf{q}}{2}\right) G_{0}\left(-\mathrm{i} \varepsilon_{n}, \mathbf{k}-\frac{\mathbf{q}}{2}\right)
$$

is the polarization operator of the electron gas [23].

The equations (17) and (18) correspond to a vicinity of the phase transition point, in which some nonzero magnetization $\mathbf{m}(\mathbf{r})$ appears.

\section{Mean field approximation}

Now we consider the possibility of realization of a nonzero uniform solution for both $\mathbf{m}(\mathbf{r})$ and $\boldsymbol{\lambda}(\mathbf{r})$ fields. This corresponds to the ferromagnetic ordering.

Assuming $\mathbf{m}$ to be constant, we obtain from equation (18) the condition of a ferromagnetic transition

$$
-\frac{c M^{2} J_{s d}^{2}}{3 T} \Pi_{0}(\mathbf{q}=0)=1
$$

For the degenerate electron gas we can ignore the weak temperature dependence of the polarization operator, and take it for $T=0[23]$

$$
\Pi_{0}(\mathbf{q})=-2 \mathrm{i} \int \frac{\mathrm{d} \varepsilon}{2 \pi} \frac{\mathrm{d}^{3} \mathbf{k}}{(2 \pi)^{3}} G_{0}\left(\varepsilon, \mathbf{k}+\frac{\mathbf{q}}{2}\right) G_{0}\left(\varepsilon, \mathbf{k}-\frac{\mathbf{q}}{2}\right) .
$$

Then, from equation (20) we obtain the critical temperature of the ferromagnetic phase transition

$$
T_{\mathrm{c}}=-\frac{c M^{2} J_{s d}^{2}}{3} \Pi_{0}(\mathbf{q}=0)
$$

which is positive (Curie temperature) provided that $\Pi_{0}(\mathbf{q}=0)<0$. 
The function $\Pi_{0}(\mathbf{q})$ corresponds to a loop diagram of the electron gas [23] and is related to the static nonlocal susceptibility of electrons $\chi_{0}(\mathbf{q})$ by the following equation

$$
\chi_{0}(\mathbf{q})=-\left(\frac{g \mu_{\mathrm{B}}}{2}\right)^{2} \Pi_{0}(\mathbf{q}),
$$

where $g$ is the Landé factor of electrons and $\mu_{\mathrm{B}}$ is the Bohr magneton. For $\mathbf{q} \rightarrow 0$ the function $-\Pi_{0}(\mathbf{q})$ tends to the electron density of states $\nu_{\mathrm{F}}$ at the Fermi level,

$$
\Pi_{0}(\mathbf{q}=0)=-\nu_{\mathrm{F}}=-\frac{m^{*} k_{\mathrm{F}}}{\pi^{2} \hbar^{2}}
$$

where $k_{\mathrm{F}}$ is the Fermi momentum. In equation (24) we take the density of states for the three-dimensional system.

\section{Ferromagnetic transition and RKKY interaction}

The self-consistency equation for magnetic polarization (16) can be analyzed in terms of RKKY interaction between the magnetic impurities. The interaction energy of two magnetic impurities with moments $\mathbf{M}^{(1)}$ and $\mathbf{M}^{(2)}$, located at a distance $\mathbf{r}$, can be presented as [17]

$$
E_{\text {int }}(\mathbf{r})=J_{s d}^{2} M_{\alpha}^{(1)} M_{\beta}^{(2)} W_{\alpha \beta}(\mathbf{r})
$$

where

$$
W_{\alpha \beta}(\mathbf{r})=-\mathrm{i} \operatorname{Tr} \int \frac{\mathrm{d} \varepsilon}{2 \pi} \frac{\mathrm{d}^{3} \mathbf{k}}{(2 \pi)^{3}} \frac{\mathrm{d}^{3} \mathbf{q}}{(2 \pi)^{3}} \mathrm{e}^{\mathrm{i} \mathbf{q} \cdot \mathbf{r}} \sigma_{\alpha} G_{0}\left(\varepsilon, \mathbf{k}+\frac{\mathbf{q}}{2}\right) \sigma_{\beta} G_{0}\left(\varepsilon, \mathbf{k}-\frac{\mathbf{q}}{2}\right) .
$$

Using equations (25) and (26), we obtain the interaction energy of two impurities with equal moments $M^{(1)}=M^{(2)}=M$ oriented ferromagnetically,

$$
E_{\mathrm{fer}}(\mathbf{r})=M^{2} J_{s d}^{2} \Pi_{0}(\mathbf{r})
$$

where the function $\Pi_{0}(\mathbf{r})$ is the Fourier transform of $\Pi_{0}(\mathbf{q})$ defined by equation (21). In terms of the pair interaction function $E_{\text {fer }}(\mathbf{r})$ we can rewrite equation (16) as

$$
\mathbf{m}(\mathbf{r})=-\frac{c}{3 T} \int \mathrm{d}^{3} \mathbf{r}^{\prime} E_{\mathrm{fer}}\left(\mathbf{r}-\mathbf{r}^{\prime}\right) \mathbf{m}\left(\mathbf{r}^{\prime}\right)
$$

Then, assuming the uniform order, we obtain the critical temperature

$$
T_{\mathrm{c}}=-\frac{c}{3} \int \mathrm{d}^{3} \mathbf{r} E_{\mathrm{fer}}(\mathbf{r})
$$

In view of (29), the critical temperature is defined by the integral of RKKY exchange interaction over all distances. 


\section{Quantum fluctuations}

The expansion of functional $F$ up to the second order in $\mathbf{m}(\mathbf{r})$ gives us the spectrum of fluctuations

$$
F=F_{0}+m_{\alpha}(\mathbf{q})\left[\frac{c M^{2} J_{s d}^{2}}{3 T} \Pi_{0}(\mathbf{q})-1\right] m_{\alpha}(-\mathbf{q}),
$$

where $F_{0}$ is a constant part, $\mathbf{m}(\mathbf{q})$ is the Fourier transform of magnetization field $\mathbf{m}(\mathbf{r})$, and the summation over vector components $\alpha$ is implied in equation (30).

Equation (30) holds for temperatures $T>T_{\mathrm{c}}$, when the constant part of magnetization is zero. Then we obtain a finite gap in the spectrum of magnetic fluctuations. It should be noted that the higher order terms of the expansion of free energy, describing the interaction of fluctuating fields, can significantly affect the mean field result. This effect should be more pronounced for low-dimensional systems.

\section{Discussion}

We have proposed a field theory approach to the problem of ferromagnetism in diluted magnetic semiconductors. It permits to analyze the ferromagnetic transition in terms of fluctuating magnetization fields. The mean field limit of the theory gives the critical temperature of ferromagnetic transition. This temperature, given by equation (29), coincides with the results obtained by other methods [4,8]. However, we can expect that the real ferromagnetic transition temperature is lower due to the strong disorder of real impurity systems as well as due to the interaction of fluctuations typical of the phase transitions within the critical region. The latter effect can be enhanced by the disorder.

We also analyzed a connection between the RKKY interaction of magnetic impurities and the mean field approximation in the framework of our formalism. The result is that an averaging over randomly distributed magnetic impurities reduces the RKKY interaction function $E_{\text {int }}(\mathbf{r})$ to a spatial integral of this function. This results in a coupling proportional to $\Pi_{0}(\mathbf{q}=0)$.

In our formalism, the saddle point equations (14) and (15), as shown in section 4, lead to the mean field result of works $[4,8]$, which also coincides with the integrated RKKY interaction approximation [24]. It should be noted, however, that a controversy exists about using the notion "mean field approximation" In particular, the approach of articles $[25,26]$ takes into account an additional mechanism of the interband coupling arising in a model of multiband electron energy spectrum. In the model of one electronic band, equation (1), the additional effect of indirect coupling is absent.

The possibility of a non-uniform magnetic ordering in magnetically doped semiconductor structures has been pointed out by Dietl et al. [27]. It was essentially imposed by a non-uniformity of the system under consideration. It should be emphasized that the phase transition in a uniform system can be also associated with a spontaneous non-uniformity of the order parameter (like the creation of striped 
structures in the case of high- $T_{\mathrm{c}}$ superconductors [28]). We can expect a tendency for a non-uniform ordering in low-dimensional systems because it is closely related (see equation 30 ) to the known properties of the polarization operator $\Pi_{0}(\mathbf{q})$ in dimensions $D=1,2,3$.

\section{Acknowledgements}

One of the authors (V.D.) is grateful to J.Barnaś for comments, to W.Dobrowolski for numerous discussions, and to the Institute of Physics, Polish Academy of Sciences, for kind hospitality. This work is partially supported by the Polish State Committee for Scientific Research through the Project 5 03B 091 20, NATO Linkage Grant No. 977615, and NATO Science fellowship CP(UN)06/B/2001/PO.

\section{References}

1. Story T., Gałązka R.R., Frankel R.B., Wolf P.A. Carrier-concentration-induced ferromagnetism in PbSnMnTe. // Phys. Rev. Lett., 1986, vol. 56, No. 7, p. 777-789.

2. de Jonge W.J.M., Story T., Swagten H.J.M., Eggenkamp P.J. Carrier-induced breakdown of ferromagnetism. // Europhys. Lett., 1992, vol. 17, No. 7, p. 631-636.

3. Eggenkamp P.J.T., Swagten H.J.P., Story T., Litvinov V.I, Swüste C.H.W., de Jonge W.J.M. Calculations of the ferromagnet-to-spin-glass transition in diluted magnetic systems with RKKY interaction. // Phys. Rev. B, 1995, vol. 51, No. 21, p. $15250-15259$.

4. Dietl T., Ohno H., Matsukura F. Hole-mediated ferromagnetism in tetrahedrally coordinated semiconductors. // Phys. Rev. B, 2001, vol. 63, No. 19, p. 195205-21.

5. Takahashi M. Electron-spin polarization in ferromagnetic semiconductors. // Phys. Rev. B, 1997, vol. 56, No. 12, p. 7389-7394.

6. Matsukura F., Ohno H., Shen A., Sugawara Y. Transport properties and origin of ferromagnetism in (Ga,Mn)As. // Phys. Rev. B, 1998, vol. 57, No. 4, p. R2037-R2040.

7. Akai H. Ferromagnetism and its stability in the diluted magnetic semiconductor (In,Mn)As. // Phys. Rev. Lett., 1998, vol. 81, No. 14, p. 3002-3005.

8. Lee B., Jungwirth T., MacDonald A.H. Theory of ferromagnetism in diluted magnetic semiconductor quantum wells. // Phys. Rev. B, 2000, vol. 61, No. 23, p. 15606-15609.

9. König J., Lin H.-H., MacDonald A.H. Theory of diluted magnetic semiconductor ferromagnetism. // Phys. Rev. Lett., 2000, vol. 84, No. 24, p. 5628-5631.

10. Litvinov V.I., Dugaev V.K. Ferromagnetism in magnetically doped III-V semiconductors. // Phys. Rev. Lett., 2001, vol. 86, No. 24, p. 5593-5596.

11. Ohno H. Making nonmagnetic semiconductors ferromagnetic. // Science, 1998, vol. 281, No. 5379, p. 951-956.

12. Dietl T., Ohno H., Matsukura F., Cibert J., Ferrand D. Zener model description of ferromagnetism in zinc-blend magnetic semiconductors. // Science, 2000, vol. 287, No. 5455 , p. $1019-1022$.

13. Ohno H., Chiba D., Matsukura F., Omiya T., Abe E, Dietl T., Ohno Y., Ohtani K. Electric-field control of ferromagnetism. // Nature, 2000, vol. 408, No. 6815, p. 944946. 
14. Ohno H., Matsukura F. A ferromagnetic III-V semiconductor: (Ga,Mn)As. // Sol. State Commun., 2001, vol. 117, No. 3, p. 179-186.

15. Dietl T. Ferromagnetic transition in diluted magnetic semiconductors. // Condens. Matter Phys., 1999, vol. 2, No. 3, p. 495-508.

16. Zener C. Interaction between the $d$ shells in the transition metals. // Phys. Rev., 1950, vol. 81 , No. 3, p. 440-444.

17. Kittel C. Indirect exchange interaction in metals. // Solid State Physics, 1968, vol. 22, p. $1-26$.

18. König J., Jungwirth T., MacDonald A.H. Theory of magnetic properties and spinwave dispersion for ferromagnetic (Ga,Mn)As. // Phys. Rev. B, 2001, vol. 64, No. 18, p. $184423-8$.

19. Berciu M., Bhatt R.N. Effects of disorder on ferromagnetism in diluted magnetic semiconductors. // Phys. Rev. Lett., 2001, vol. 87, No. 10, p. 107203-4.

20. Chudnovskiy A.L., Pfannkuche D. Influence of disorder on ferromagnetism in diluted magnetic semiconductors. // Phys. Rev. B, 2002, vol. 65, No. 16, p. 165216-7.

21. Fradkin E. Field Theories in Condensed Matter Systems. Redwood City, AddisonWesley, 1991.

22. Efetov K.B. Supersymmetry in Disorder and Chaos. London, Cambridge University Press, 1997.

23. Abrikosov A.A., Gorkov L.P., Dzyaloshinski D.E. Methods of Quantum Field Theory in Statistical Physics. New York, Dover, 1963.

24. Dietl T., Haury A., d'Aubigné Y.M. Free carrier-induced ferromagnetism in structures of diluted magnetic semiconductors. // Phys. Rev. B, 1997, vol. 55, No. 6, p. R3347R3350.

25. Semenov Yu.G., Ryabchenko S.M. On the theory of carrier-induced ferromagnetism in diluted magnetic semiconductors. // Low Temp. Physics, 2000, vol. 26, No. 12, p. $886-889$.

26. Semenov Yu.G., Ryabchenko S.M. Exactly solvable model for carrier-induced paramagnetic-ferromagnetic phase transition in diluted magnetic semiconductors. // Physica E, 2001, vol. 10, No. 1-3, p. 165-169.

27. Dietl T., Ohno H. Ferromagnetism in III-V and II-VI semiconductor structures. // Physica E, 2001, vol. 9, No. 1, p. 185-193.

28. Rigamonti A., Borsa F., Carretta P. Basic aspects and main results of NMR-NQR spectroscopies in high-temperature superconductors. // Rep. Prog. Phys., 1998, vol. 61, No. 10 , p. $1367-1439$. 


\title{
Феромагнітне впорядкування в легованих магнітних напівпровідниках
}

\author{
А.Г.Слободський ${ }^{1,2,3}$, В.К.Дугаєв ${ }^{1,4}$, М.Віейра ${ }^{4}$
}

1 Інститут проблем матеріалознавства,

Національна Академія наук України,

58001 Чернівці, вул. І. Вільде, 5

2 Інститут фізики, Польська Академія наук,

Польща 02-668 Варшава, ал. Льотнікув, 32/46

3 Фізичний інститут, Університет Вюрцбурґ, Хубланд, Німеччина, 97074 Вюрцбург

4 Факультет електроніки та звязку,

Вища інженерна школа у Лісабоні,

Португалія, 1949-014 Лісабон

Отримано 22 листопада 2001 р., в остаточному вигляді 25 червня 2002 p.

Запропоновано загальний підхід до проблеми феромагнітного фазового переходу в легованих магнітних напівпровідниках. Температуру Кюрі феромагнітного переходу обчислено в наближенні середнього поля. Показано, що температура Кюрі визначається через інтеграл від парної взаємодії між магнітними домішками.

Ключові слова: магнітні напівпровідники, магнітні домішки, феромагнетизм

PACS: $75.30 .-m, 75.50 . D d, 75.50 . P p$ 